\title{
CASE REPORT - SEPTATE GALL BLADDER
}

Ankur Prakash¹, R. C. Jain², V. K. Singh ${ }^{3}$, Mayank Mathur ${ }^{4}$

\section{HOW TO CITE THIS ARTICLE:}

Ankur Prakash, R.C. Jain, V.K. Singh, Mayank Mathur. "Case Report- Septate Gall Bladder". Journal of Evolution of Medical and Dental Sciences 2014; Vol. 3, Issue 06, February 10; Page: 1320-1322,

DOI: $10.14260 /$ jemds/2014/1986

ABSTRACT: The anatomy facing a surgeon during cholecystectomy is challenging as it involves complex relationship between the gallbladder, hepatic artery and extra-hepatic biliary tree. We report a case of septate gall bladder which was successfully treated with laparoscopic cholecystectomy. Lack of awareness, non-specific symptoms, signs and inadequacy of imaging methods are possible reasons for the reported problem of overlooking of this entity. Complete identification and removal of gallbladder is mandatory, as a remnant may result in recurrence of symptoms or stones.

KEYWORDS: Gallbladder anomalies, laparoscopic treatment, septate gallbladder.

INTRODUCTION: Congenital anomalies of the gallbladder are rare and wide ranges of malformations pertaining to all aspects have been described. ${ }^{1}$ Septate gallbladder has not been well documented because it is usually asymptomatic or discovered accidentally during the evaluation of abdominal pain. ${ }^{1}$ Rarely due to septation there may be stone formation, which might lead to recurrent abdominal pain. ${ }^{2}$ We report a case of septate gallbladder that underwent successful laparoscopic cholecystectomy (LC). This case illustrates the need for complete removal of gallbladder during surgery. Precise intraoperative recognition of vascular and biliary anatomy is necessary to avoid common bile duct (CBD) injuries and gallbladder remnants.

CASE REPORT: A 44-year-old female patient was admitted to our hospital with a right upper quadrant pain recurring for the past 2 months. There was a history of postprandial fullness and belching. There was no history of jaundice in past. His blood values were within normal range. Ultrasound (US) of upper abdomen showed the gallbladder filled with multiple calculi. Laparoscopic Cholecystectomy was planned.

At laparoscopy, flimsy adhesions were identified at the fundus of the gallbladder. After retracting the fundus towards the ipsilateral shoulder dense adhesions were present at the body of the gallbladder. After completing adhesiolysis with blunt and sharp dissection, Calot's triangle was identified. Cystic artery and Cystic duct were clipped and divided. Laparoscopic cholecystectomy was successfully completed without any complication. The postoperative period was uneventful and the patient was discharged on the second postoperative day. On follow-up appointments, patient was in good condition. 


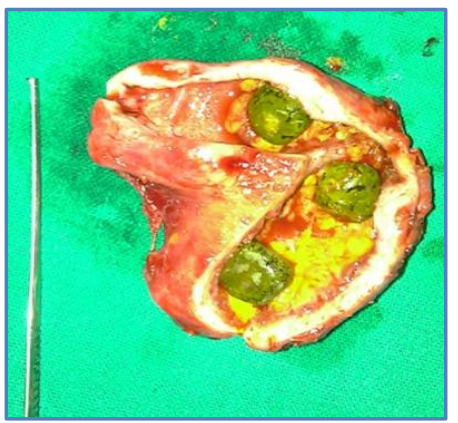

Gross examination of the specimen showed a vertical septum. There was evidence of acute inflammatory changes over entire wall

DISCUSSION: Anomalies of the gallbladder have been classified into malformation of shape, number, site, size. These anomalies may be asymptomatic or may cause wide range of complications.

Septate gallbladder is characterized by the presence of a septum that divides the gallbladder in two chambers. When the septum dividing the gallbladder lies longitudinally it is called bilobed gallbladder and when there is a transverse septum separating the fundus from the rest of the gallbladder it is called an hour-glass gallbladder ${ }^{2}$.In our case it was bilobed gall bladder. Septate gallbladders most likely result from incomplete resolution of the solid stage of gallbladder development that is present before the third month of fetal development. These gallbladder septae are most commonly single, but multi septate gallbladder, as well as post inflammatory adhesions and compartmentalization of the gallbladder have also been described. ${ }^{3}$

In our patient the septa were most probably congenital in origin with superadded inflammation which gave rise to classic septate shaped gallbladder. Secondly bile stasis and infection would have led to the development of the gallstones in this patient.

According the Boyden classification, ${ }^{4}$ these congenital anomalies of gallbladder are classified according to the ductal formation as bilobed (or bifid), "V-shaped" and "H-shaped" (or ductular) gallbladder types. Classification of different types of double gallbladders is shown in Table 15 . To date US oral cholecystography oral cholecysto-computed tomography, scintigraphy, magnetic resonance cholangiography, percutaneous transhepatic cholangiography and endoscopic retrograde cholangiopancreatography have been used preoperatively to diagnose these anomalies ${ }^{6}$.

A. One cystic duct entering the common bile duct:

Gall Bladder Septum (no evidence of duplication on the surface)

Division of the gallbladder into two lobes joining at the neck to form a normal cystic duct

(V duplication)

Complete separation of the gallbladders, each with its own cystic ducts to join to form a common cystic (Y duplication)

B. Two cystic ducts separately entering the bile ducts:

Two cystic ducts entering the common bile ducts ( $\mathrm{H}$ - duplication)

One cystic duct entering the common bile duct, the other entering the right or left hepatic duct.

Table 1: Classification of different types of double gallbladder ${ }^{5}$ 
Consequently, identification and definition of biliary anatomy is mandatory to prevent biliary system injury. Injury to the ductal system usually occurs during dissection of Calot's triangle. All connective tissue and fat must be removed completely to clearly expose the junction of the cystic duct with the gallbladder. We also performed meticulous dissection, keeping in mind principles of LC to prevent inadvertent injury. A careful appraisal of reported literature clearly emphasizes the need for removal of complete gallbladder to prevent surgical complications and repeated explorations.

In conclusion, this case illustrates the need for complete removal of gallbladder, as a remnant could be a good seat for development of stone in future and subsequent recurrence of symptoms.

\section{REFERENCES:}

1. Gross RE. Congenital anomalies of the gall bladder: A review of 148 cases, with report of double gall bladder. Arch Surg. 1936; 32:131-62.

2. Flannery MG, Caster MP. Congenital hour-glass gall bladder. South Med J. 1957; 50:1255-8. [PubMed].

3. Adear H, Barki Y. Multi septate gall bladder in a child: Incidental diagnosis on sonography. Paediatr Radiol. 1990; 20:192. [PubMed].

4. Gigot J, Van Beers B, Goncette L. Laparoscopic treatment of gallbladder duplication: A plea for removal of both gallbladders. Surg Endosc. 1997; 11:479-82. [PubMed].

5. Silvis $\mathrm{R}$, van Wieringen $\mathrm{AJ}$, van der Werken $\mathrm{CH}$. Reoperation for a symptomatic double gallbladder. Surg Endosc. 1996; 10:336-7. [PubMed].

6. Oyar O, Yesildag A, Gulsoy U, Sengul N, Isler M. Bilobed gallbladder diagnosed by oral cholecysto-CT. Comput Med Imaging Graph. 2003; 27:315-9. [PubMed].

\section{AUTHORS:}

1. Ankur Prakash

2. R. C. Jain

3. V. K. Singh

4. Mayank Mathur

\section{PARTICULARS OF CONTRIBUTORS:}

1. Junior Resident - Third Year, Department of General Surgery, Eras Lucknow Medical College and Hospital.

2. Professor, Department of General Surgery, Eras Lucknow Medical College and Hospital.

3. Senior Resident, Department of General Surgery, Eras Lucknow Medical College and Hospital.
4. Junior Resident - Second Year, Department of General Surgery, Eras Lucknow Medical College and Hospital.

\section{NAME ADDRESS EMAIL ID OF THE CORRESPONDING AUTHOR:}

Dr. Ankur Prakash,

JR-3, Department of General Surgery,

Eras Lucknow Medical College and Hospital.

E-mail: ankurprakash2@gmail.com

Date of Submission: 13/01/2014.

Date of Peer Review: 14/01/2014.

Date of Acceptance: 25/01/2014.

Date of Publishing: 04/02/2014. 